\title{
Correction of Bilateral Tessier No. 2, 3, and 12 Facial Cleft with Anopthalmia
}

\author{
Seong-Yong Moon, Seong-Gon Kim¹, Young-Ju Park², Young-Wook Park ${ }^{1}$ \\ Department of Oral and Maxillofacial Surgery, College of Dentistry, Chosun University, ${ }^{1}$ Department of Oral and Maxillofacial Surgery, \\ College of Dentistry, Gangneung-Wonju National University, ${ }^{2}$ Department of Oral and Maxillofacial Surgery, \\ Kangnam Sacred Heart Hospital, College of Medicine, Hallym University
}

\begin{abstract}
Oblique facial cleft is a rare congenital deformity. Its incidence has been reported as $0.24 \%$ of all reported cases of facial cleft. We report on a patient who had a left-sided oblique facial cleft with anopthamia, including lip and palate, nose alar base, and medial canthus. The patient also had a right-sided oblique facial cleft, which included lip and palate, nose alar base, medial canthus, and upper eye brow. Primary closure of the facial cleft was performed using multiple Z-plasty after excision of scar tissue.
\end{abstract}

Key words: Oblique facial cleft, Anopthamia

\section{Introduction}

Craniofacial cleft is an extremely rare disease, with a prevalence rate of approximately $0.24 \%$ in all reported cases of facial cleft[1]. The incidence of facial cleft was reported as 1.43 4.85 out of 100,000 newborn infants[2]. Tessier's classification, reported in 1976, has been the representative system for classification of facial clefts. Its classification is based on anatomical position. Tessier's classification of facial cleft types ranges from 0 to 14 , based on the central line, which connects the sagittal plane and both orbital fossa[3]. Fearon suggested another classification system for use in deciding on the treatment method; using this system, facial clefts are classified according to four types, depending on anatomical position[4].

In the embryo, facial development occurs over a period of $4 \sim 8$ weeks. Craniofacial cleft is created by failure of fusion of mesoderm of the facial area during the state of embryonic development. An oblique facial cleft is formed by extraordinary fusion of the lateral nasal process and maxillary process. Oblique facial cleft may appear without relationship to other syndromes, and there is no sexual difference[1].

We experienced a case of bilateral Tessier No. 2, 3, and 12 facial cleft with anopthalmia. This patient was treated with primary closure of the facial cleft, performed using multiple Z-plasty after excision of scar tissue. 


\section{Case Report}

The patient, a three-year-old female without specific systemic disease, was found by a medical volunteer who was visiting the Philippines. The cleft on the right side of her face was classified as Tessier type 2, defined as a facial cleft running from the upper lip to the ala of the nose and from the eyebrow of the upper eyelid to the forehead, and Tessier type 12, defined as an independent cranial cleft. On the left side, her eyeball could not be seen and the lower eyelid was dislocated toward the latero-down direction. The nasolacrimal duct was obstructed and the oblique facial cleft extended from the medial canthus through the ala of the nose to the cleft lip and palate. In addition, a severe Tessier type 3 facial cleft with anophthalmia was observed on the left side (Fig. 1). According to Fearon's classification, a paramedian cleft (type II) was observed on both the left and right sides.

Defect of palate, alveolar bone, medial and anterior wall of the left maxilla, and medial and inferior wall of orbit were observed on computerized tomography images (Fig. 2). Surgery performed on this patient focused on removal of all of visible scars and primary closure of the facial clefts using multiple Z-plasty. Through dissection of the inferior of left orbit and cheek, sound tissue was drawn to the ala of the nose and then sutured in layers. For correction of the right ala of the nose, Z-plasty was performed at the medial canthus and lower eyelid. For correction of the left nasal ala and lower eyelid, Z-plasty was also performed at the medial canthus and lower eyelid. Straight line closure was performed at the left nasal ala and upper lip, where a broad defect was observed (Fig. 3). No procedure for correction of bony defects was performed. There was no postoperative complication. One year after the operation, the patient's facial appearance was acceptable (Fig. 4).

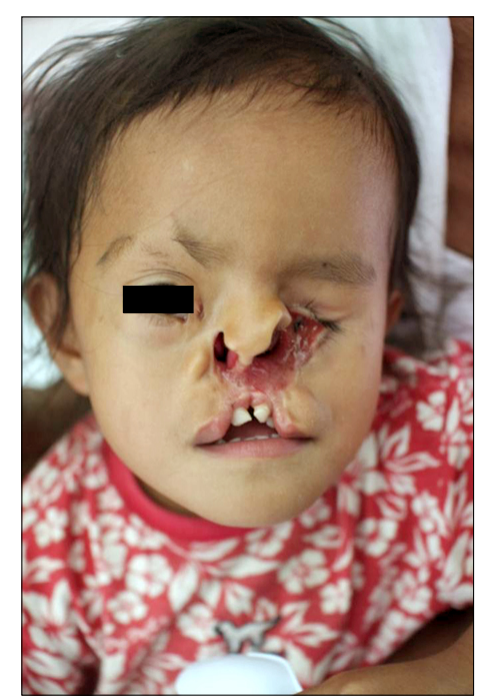

Fig. 1. Preoperative photograph. Right facial cleft is classified as Tessier No. 2 and 12, left facial cleft is classified as Tessier No. 3.
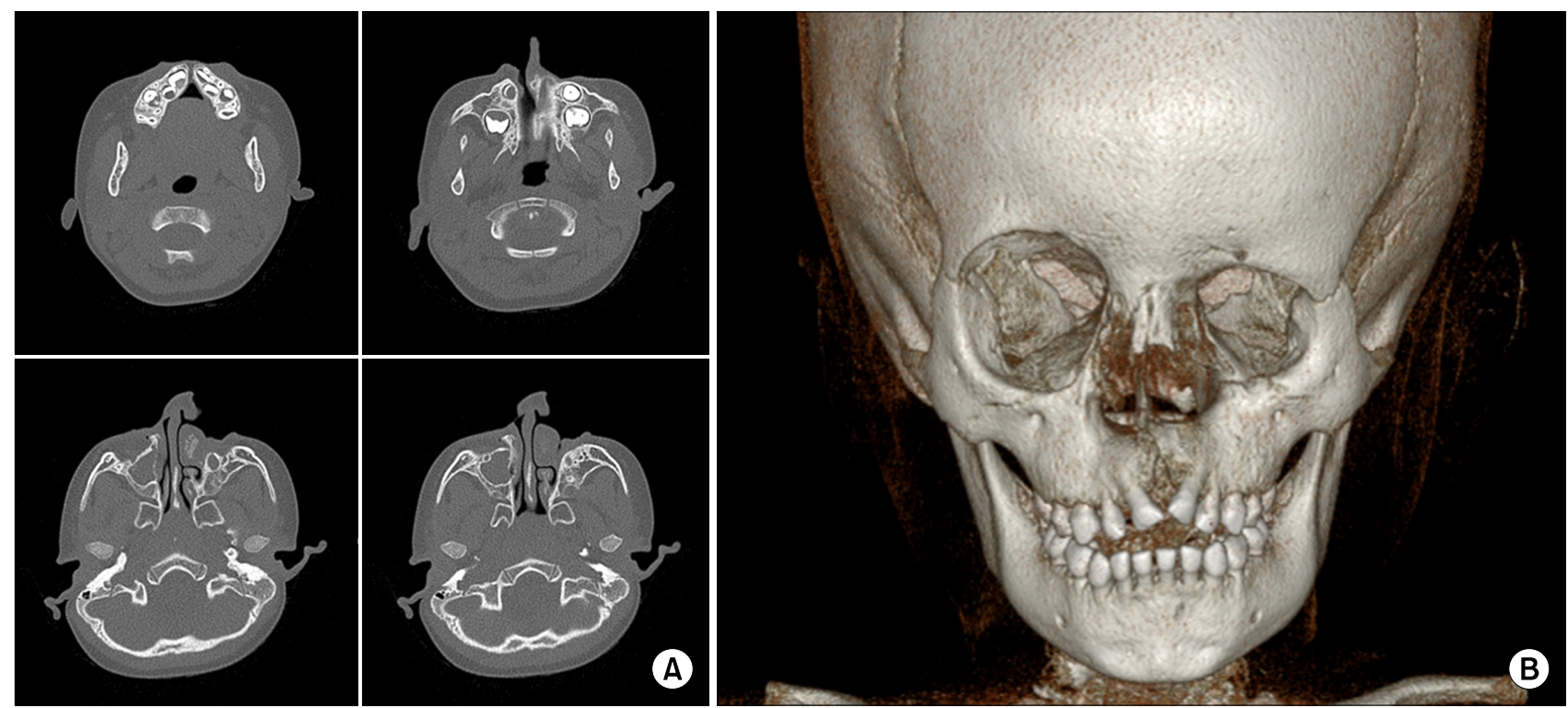

Fig. 2. (A) Axial computed tomography (CT) images. (B) Three-dimensional reconstruction from CT images. Bone defect was observed on alveolus, palate, left medial, and anterior wall of the maxilla, and orbital rim. 


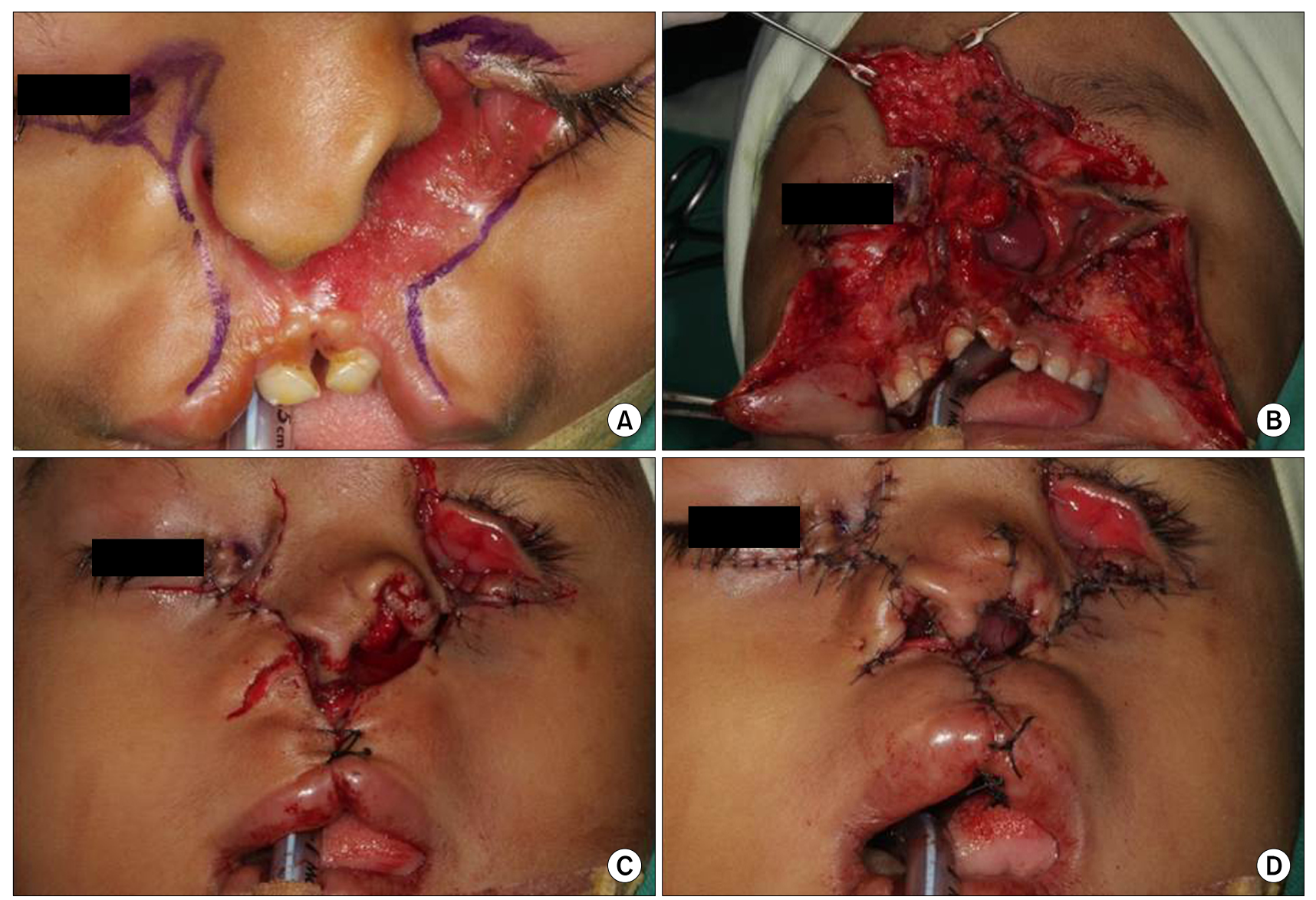

Fig. 3. Intraoperative photographs. (A) Incision design. (B, C) Multiple Z-plasty after excision of scar tissue. (D) Primary closure of facial cleft.

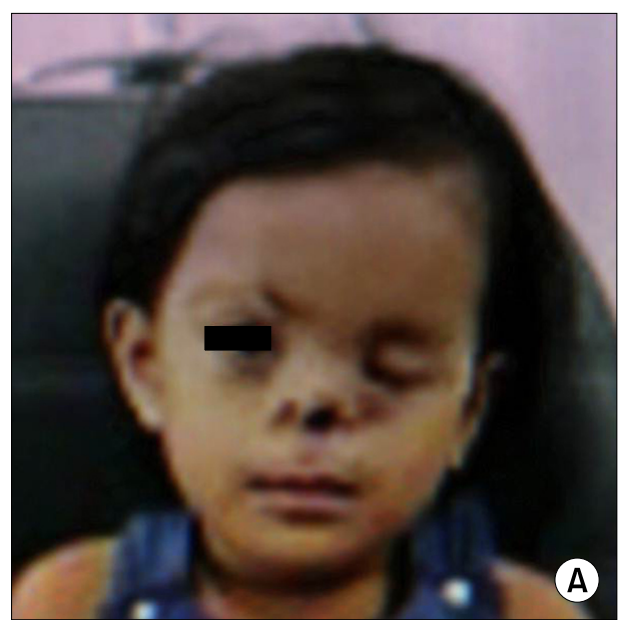

\section{Discussion}

Craniofacial cleft is an extremely rare disease. Tessier's classification, which has been widely used for treatment of facial clefts, is based on two lines connecting the mid-sagittal plane and a center-line of the orbit fossa ac-

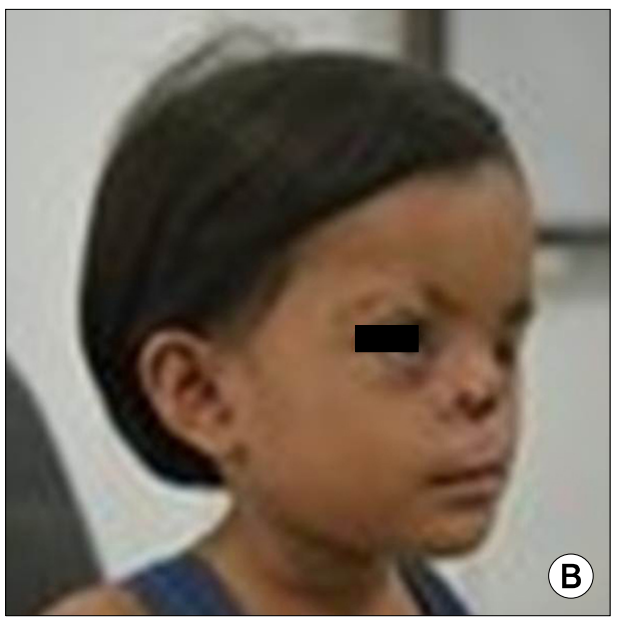

Fig. 4. Postoperative photographs one-year after the operation. (A) Frontal view. (B) Lateral view. 
medial canthus was observed.

The incidence of Tessier type 2 facial cleft was reported as 23 out of 345 cases[5]. This type of patient exhibits unique characteristics of soft tissue and hard tissue, different from those of Tessier type 1 or 3 . The most distinctive feature of Tessier type 2 is a V-shaped incisura after deformation of the middle third on the soft tissue of the nostril border. In addition, the lateral area of the nose on the morbid side is flat and the nasal bridge is wider. On hard tissue, the nasal septum is preserved, but shows deviation because of the deformity of surrounding tissues. Tessier type 12 is the cranial part cleft, which acts as a proxy for Tessier type 2 . The inner margin of the eyebrow becomes deformed as a result of passing between the frontal process of the maxilla and the nasal bone.

Tessier type 3 facial cleft is accompanied by a cleft lip and palate. It extends from the upper lip to the ala of the nose, lower eyelid, and inner canthus, and shows defects of soft and hard tissue at the same time. Stoll et al.[6] reported three causes of anophthalmia. The first is anophthalmia due to failure of optic outgrowth in the forebrain, the second is anophthalmia due to malformation in the anterior end of the forebrain, and the third is anophthalmia due to degeneration arising from the optic vesicle and tract. The third form usually appears closer to microphthalmia, rather than real anophthalmia[7]. This case can be considered as the third form.

Due to the difficulty of antenatal screening, severe facial dysmorphia is generally diagnosed too late, or after delivery[8]. The usefulness of three-dimensional ultrasonography, rather than two-dimensional, in diagnosis of an antenatal facial cleft has been reported[9]. Radiological examination is also necessary for diagnosis and evaluation of a facial cleft. Axial and three-dimensional computed tomography can provide information about soft and hard tissue defects of facial cleft patients[10].

The ultimate goal of treatment of facial cleft is the functional correction of macrosomia, the soft tissue reconstruction of palpebra for protection of eyeballs, proper separation of oral, nasal, and orbital cavities, and esthetic correction of facial appearance[11]. Tissue expander, advancement and rotation flap, autogeneous bone graft, and multiple Z-plasty have generally been used for correction of facial clefts[12]. The operation time for bone graft for treatment of bone defect has been controversial in facial cleft patients. Resnick and Kawamoto[13] reported a preference for bone grafting before the age of five. However, premature bone graft could cause significant bone resorption and interruption of normal bone remodeling[14].

For correction of a Tessier type 2 facial cleft, the lateral side of the nose can be used as a donor site for the full thickness rotation flap[15]. However, an asymmetrical nostril or a large defect after the primary repair may require secondary correction. Severe facial cleft including the entire nose can be corrected with a longer rotation flap or a forehead flap. Tessier type 3 defect can generally be corrected using a multiple cheek flap or a nasal flap. Nasal flap cannot be used for large defects on the lateral nasal surface, because tension of soft tissue is too great when correction is performed using a nasal flap. To overcome such tissue insufficiency, Toth et al.[16] suggested using a tissue expander on the forehead. However, due to scar formation on the forehead, straight-line closure was used more often than a tissue expander. In our patient, multiple Z-plasty was performed at the medial canthus and lateral nasal surface for correction of the right nasal area. On the left side, straight-line closure was performed on the lateral surface of the nose, and multiple Z-plasty was performed for correction of the medial canthus and lower eyelid cleft.

Correction of both sides of the facial cleft is difficult. In the case of a midline facial cleft (Type I), correction of telorbitism is needed. In the case of a median facial cleft (Type II) and orbital cleft (Type III), the unilateral nose and lacrimal system should be restored and an eyelid flap should be used to cover the eyeball, respectively. Correction of a cleft lip and palate, and secondary bone graft are also needed in Type II and III cases. However, satisfactory performance of all of these procedures at one time is difficult, and repeated operations are needed.

The focus of treatment of our patient was scar excision and primary closure of each part of the bilateral facial cleft. The patient's appearance has shown considerable improvement one year after the operation. However, the length of the follow-up period has been limited. In the future, scar revision, bone graft, and orthodontic treatment will be needed in this patient. 


\section{References}

1. Eppley BL, van Aalst JA, Robey A, Havlik RJ, Sadove AM. The spectrum of orofacial clefting. Plast Reconstr Surg 2005; 115:101e-14e.

2. Kawamoto HK Jr. The kaleidoscopic world of rare craniofacial clefts: order out of chaos (Tessier classification). Clin Plast Surg 1976;3:529-72.

3. Tessier P. Anatomical classification facial, cranio-facial and latero-facial clefts. J Maxillofac Surg 1976;4:69-92.

4. Fearon JA. Rare craniofacial clefts: a surgical classification. J Craniofac Surg 2008;19:110-2.

5. Ortiz Monasterio F, Fuente del Campo A, Dimopulos A. Nasal clefts. Ann Plast Surg 1987;18:377-97.

6. Stoll C, Alembik Y, Dott B, Roth MP. Congenital eye malformations in 212,479 consecutive births. Ann Genet 1997;40:122-8.

7. Wenbin Z, Hanjiang W, Xiaoli C, Zhonglin L. Tessier 3 cleft with clinical anophthalmia: two case reports and a review of the literature. Cleft Palate Craniofac J 2007;44:102-5.

8. Gawrych E, Janiszewska-Olszowska J, Walecka A, Syryńska M, Chojnacka H. Lobar holoprosencephaly with a median cleft: case report. Cleft Palate Craniofac J 2009;46:549-54.

9. Tonni G, Centini G, Rosignoli L. Prenatal screening for fetal face and clefting in a prospective study on low-risk population: can 3- and 4-dimensional ultrasound enhance visualization and detection rate? Oral Surg Oral Med Oral Pathol Oral Radiol Endod 2005;100:420-6.

10. Galante G, Dado DV. The Tessier number 5 cleft: a report of two cases and a review of the literature. Plast Reconstr Surg 1991;88:131-5.

11. Hunt JA, Hobar PC. Common craniofacial anomalies: facial clefts and encephaloceles. Plast Reconstr Surg 2003;112:606-15.

12. Aköz T, Erdoğan B, Görgü M, Kutlay R, Dağ F. Bilaterally involved Tessier No. 4 cleft: case report. Cleft Palate Craniofac J 1996;33:252-4.

13. Resnick JI, Kawamoto HK Jr. Rare craniofacial clefts: Tessier no. 4 clefts. Plast Reconstr Surg 1990;85:843-9.

14. van der Wal KG, van der Meulen BD, van der Biezen JJ, Mulder JW. Bone grafting the piriform aperture deformity in isolated cleft lip patients: indication, technique, and results. J Oral Maxillofac Surg 1997;55:1089-93.

15. Agrawal K, Panda KN, Prasad S. Isolated Tessier no. 1 cleft of the nose. Ann Plast Surg 1998;41:311-3.

16. Toth BA, Glafkides MC, Wandel A. The role of tissue expansion in the treatment of atypical facial clefting. Plast Reconstr Surg 1990;86:119-22. 